\title{
A controlled trial of 6-weeks' treatment with a novel inhaled phosphodiesterase type-4 inhibitor in COPD
}

\author{
J. Vestbo*,\#, L. Tan ${ }^{\star}$, G. Atkinson` and J. Ward", on behalf of the UK-500,001 Global \\ Study Team ${ }^{+}$
}

ABSTRACT: Anti-inflammatory drugs are lacking in chronic obstructive pulmonary disease (COPD) and inhibitors of the phosphodiesterase type-4 (PDE4) enzyme have been suggested to be an interesting class of drugs to treat inflammation in COPD. The present authors report the findings of a phase II trial of a novel inhaled PDE4 inhibitor.

Three doses, $0.1,0.4$ and $1.0 \mathrm{mg}$ b.i.d., of the compound UK-500,001 were tested in a doubleblind, placebo-controlled, 6-week trial in 209 patients with moderate-to-severe COPD. The primary efficacy parameter was trough forced expiratory volume in one second after 6 weeks of treatment, and secondary end-points included other lung function end-points and symptom scores assessed at 2-week intervals. The present study was stopped following a planned interim analysis for futility.

No effect on the primary efficacy parameter, other measures of lung function or symptom scores was observed at any dose of UK-500,001 after 6 weeks of treatment. However, after the first 2 weeks of treatment, an improvement in a number of outcome measures in the $1.0 \mathrm{mg} \mathrm{b.i.d.}$ dose group was observed compared with placebo. The drug was well tolerated, although PDE4 inhibitor-related side-effects were observed, especially in the highest dose group.

The findings of the present study question the role of inhaled phosphodiesterase type- 4 inhibitors in chronic obstructive pulmonary disease.

KEYWORDS: Chronic obstructive pulmonary disease, inhaled medication, phosphodiesterase type-4-inhibitors, treatment

hronic obstructive pulmonary disease (COPD) is an inflammatory disease affecting the airways and, in the majority of cases, is caused by tobacco smoking [1]. Inhibitors of the phosphodiesterase type-4 (PDE4) enzymehave been developed as anti-inflammatory agents for use in COPD. It is hypothesised that selective PDE4 inhibition will improve lung function by attenuating the production of inflammatory mediators that are important in the pathophysiology of COPD. Trials of oral formulations of cilomilast and roflumilast have shown clinical efficacy, evidenced by improvements in trough forced expiratory volume in one second (FEV1) in studies of $\geqslant 6$ weeks' duration, but dosing has been limited by PDE4-mediated side-effects, especially those affecting the gastrointestinal tract [2-4]. UK-500,001 is an isoform-selective, subtype-nonselective (PDE4A median inhibitory concentration (IC50) 1.9 nM, PDE4B IC50 $1.01 \mathrm{nM}$ and PDE4D IC50 $3.78 \mathrm{nM})$ PDE4 inhibitor developed as an inhaled anti-inflammatory agent for the maintenance treatment of COPD. UK-500,001 has been developed as a dry powder for inhalation (DPI), with the premise that topical administration of a PDE4 inhibitor to the lung may reduce the propensity for side-effects while maintaining equivalent or possibly introducing even greater levels of clinical efficacy than those observed with oral agents. In vitro studies have shown the compound to effectively inhibit mediator release from human isolated neutrophils and peripheral blood mononuclear cells; UK-500,001 was similar to roflumilast in terms of PDE4 enzyme potency and whole-cell mediator release assays [5]. In animal studies, inhaled UK-500,001 inhibited lipopolysaccharide-induced neutrophilia and tumour necrosis factor- $\alpha$ accumulation in bronchoalveolar lavage fluid in rats in a dose-dependent manner. Also, the compound inhibited histamine-induced bronchoconstriction in anaesthetised rabbits and guineapigs, the latter in a dose-dependent manner [6].
AFFILIATIONS

*North West Lung Centre, Wythenshawe Hospital/University of Manchester,

${ }^{\#}$ Medicines Evaluation Unit

Manchester,

"Clinical R\&D, Pfizer Inc., Sandwich, UK.

+For a full list of contributors please see the Acknowledgements section.

CORRESPONDENCE

J. Vestbo

North West Lung Centre

Wythenshawe Hospital

Southmoor Road

Manchester M23 9LT UK

Fax: 441612915730

E-mail: Jorgen.vestbo@

manchester.ac.uk

Received:

May 042008

Accepted after revision:

January 062009

CLINICAL TRIAL

The present study is registered on the publicly accessible database www.clinicaltrials.gov (NCT00263874).

STATEMENT OF INTEREST Statements of interest for all authors and the study itself can be found at www.erj.ersjournals.co.uk/misc/ statements.dtl 
The primary purpose of the present study was to evaluate the efficacy and safety/tolerability of UK-500,001 DPI in patients with COPD. The doses in the present study were intended to explore the dose range in anticipation of subsequent largerscale efficacy and tolerability studies. The maximum dose (1.0 mg b.i.d.) was selected based on the maximum tolerated dose (MTD) determined in phase I clinical studies, adjusted due to pharmacokinetic differences observed in smokers and elderly subjects; and the lower doses were selected based on those predicted to be efficacious in pre-clinical in vivo models.

\section{METHODS}

\section{Study design}

The present study was a multicentred, 6-week, randomised, double-blind, placebo-controlled, parallel group study in adult patients with moderate-to-severe COPD, as defined by Global Initiative for Chronic Obstructive Lung Disease (GOLD) stages II-III. The study comprised eight clinic visits, which included the following: one screening visit, two visits during the run-in phase (weeks 2 and 1), a baseline visit with randomisation (week 0) at the start of the double-blind treatment phase, three visits during the double-blind treatment phase (weeks 2, 4 and 6) and, finally, one follow-up visit (week 8) following a 2-week washout (run-out) phase. The present study is registered on the publicly accessible database www.clinicaltrials.gov (NCT00263874).

\section{Study subjects}

Inclusion criteria were as follows: age 40-80 yrs; a smoking history of $\geqslant 10$ pack-yrs; a body mass index $<35 \mathrm{~kg} \cdot \mathrm{m}^{-2}$ and total body weight $>40 \mathrm{~kg}$; moderate-to-severe COPD according to GOLD criteria for $\geqslant 6$ months, with stable disease for $\geqslant 1$ month prior to screening; and informed written consent.

Exclusion criteria included: a history of more than two exacerbations of COPD requiring treatment with oral steroids in the preceding year; hospitalisation for the treatment of COPD within 3 months of screening, or more than twice during the preceding year; or a history of a lower respiratory tract infection or significant disease instability during the month preceding study entry.

Other exclusion criteria were adult asthma, other chronic respiratory disorders, a history or presence of respiratory failure, cor pulmonale or right ventricular failure, or home oxygen therapy.

\section{Study treatment}

At the beginning of the double-blind treatment phase (week 0), patients were randomised to receive either UK-500,001 (Pfizer Ltd, Sandwich, Kent, UK) DPI (0.1, 0.4 or $1.0 \mathrm{mg}$ b.i.d.) or matching placebo delivered via a single-pin monodose capsule inhaler device (model 5; manufactured by Plastiape SpA, Osnago, Italy and supplied by Miat SpA, Milan, Italy). An MTD approach was used for dose selection. Phase I studies indicated that $2.0 \mathrm{mg}$ b.i.d. was the MTD in healthy volunteer studies; however, a study in smokers and elderly patients showed modestly increased systemic exposure. Given this modest increase in smokers and elderly subjects, both of which are characteristics of COPD, the maximum dose of $1.0 \mathrm{mg}$ b.i.d. was selected. Patients were randomised to the treatment groups according to the following ratio: $0.1 \mathrm{mg}$ b.i.d./0.4 $\mathrm{mg}$ b.i.d./1.0 $\mathrm{mg}$ b.i.d./placebo 1:1:1:1.
Patients used ipratropium bromide metered-dose inhaler (MDI) $40 \mu \mathrm{g}$ q.i.d. as maintenance therapy and salbutamol MDI for rescue use throughout the study. No other COPD medications were allowed.

\section{Study end-points}

The primary end-point was considered to be the mean change from baseline in trough FEV1 at week 6. Pulmonary function was measured at each visit according to American Thoracic Society standards [7] using the same spirometer (Masterscope $\mathrm{CT}$; Viasys Healthcare $\mathrm{GmbH}$, Hoechberg, Germany) at each centre. (Centres were from Argentina, Australia, Canada, Chile, Croatia, Czech Republic, Hungary, Spain and the UK; all investigators and countries are listed in the Acknowledgements section). Measurements were made before the use of study medication and bronchodilators; in addition, measurements were made after inhaling $200 \mu \mathrm{g}$ of salbutamol at baseline and at the end of the study. Centralised over-read/ interpretation was performed for all lung function parameters to ensure consistent quality and reproducibility.

Secondary end-points were other spirometric measures (forced vital capacity (FVC), FEV6), inspiratory capacity (IC) and peak expiratory flow rate at weeks 2, 4, 6 and 8, Mahler dyspnoea index (baseline dyspnoea index/transitional dyspnoea index (BDI/TDI)) at weeks 2, 4 and 6, COPD symptoms (breathlessness, sputum production and cough) and bronchodilator use recorded daily by the patient and Global Impression of Change (recorded by both clinician and patient) at the end of the study. Adverse events (AEs) were recorded at each study visit, as well as ECG and laboratory data, and vital signs.

\section{Interim analysis}

A pre-specified interim analysis for efficacy was performed based on the primary efficacy parameter. This analysis was triggered when 66 patients in the $1.0 \mathrm{mg}$ and placebo dose groups had completed double-blind treatment. The analysis compared the $1.0 \mathrm{mg}$ b.i.d. and placebo-treated groups only, using a group sequential approach. The present study could only be stopped for futility at the interim analysis, and not for efficacy. If futility was not triggered, the study could complete recruitment and proceed to a final analysis.

\section{Sample size determination}

The sample size calculation was based on the primary efficacy end-point, and the study was designed using group sequential methodology, allowing for an interim analysis for futility (comparing the $1.0 \mathrm{mg}$ b.i.d. dose group and placebo). Assuming a true difference between the $1.0 \mathrm{mg}$ b.i.d. treatment group and a placebo of $0.0 \mathrm{~mL}$, then the study was designed such that the probability of stopping the study for futility was $\sim 70 \%$, based on a one-sided 5\% level significance test and a Pocock stopping boundary. Furthermore, if the study was not stopped at the interim, it was required that the final analysis should have at least an $80 \%$ power to detect a difference in mean change from baseline in trough FEV1 at week 6 of $75 \mathrm{~mL}$, based on a one-sided 5\% significance level. The present study was designed with an estimated SD of $114 \mathrm{~mL}$, which resulted in a planned interim after 18 patients had completed in each treatment group, with a final study size of 200 randomised patients. However, emerging data from other studies suggested 
that the SD estimate may have been too low, so a blinded sample size re-estimation was performed after 30 patients had completed double-blind treatment. This resulted in a revised SD estimate of $173 \mathrm{~mL}$. In order to retain the statistical properties of the present study as outlined, the subject numbers were revised with a planned interim analysis after 33 patients had completed in each treatment group. If the study did not stop at the interim analysis, the final study size was correspondingly increased to 324 patients. A drop-out rate of $\leqslant 11 \%$ was assumed throughout.

Patients with a baseline value and at least one "on-treatment" value recorded were included in the analyses.

\section{RESULTS}

A total of 209 of an intended 324 patients, 152 males and 57 females, were entered into the study at 26 clinical centres (fig. 1). The pre-specified interim analysis for efficacy was performed based on the primary efficacy end-point as mentioned above. The analysis was performed after 150 patients had completed double-blind treatment and the study was stopped for futility at this analysis. There was no adverse safety signal and patients who were already in the doubleblind phase of the study were informed of these findings and could either withdraw or continue to participate in the study to completion. Therefore, the present results reflect the final analysis for all 209 patients entered in the trial, and not those obtained at the interim analysis for futility.
Table 1 shows the characteristics of all randomised subjects in each treatment group. These data confirm that the subjects recruited into the trial were indeed GOLD stage II-III patients with a baseline post-bronchodilator FEV1 of $\sim 50 \%$ of predicted in each group, and a mean reversibility (to $400 \mu \mathrm{g}$ salbutamol) of $\sim 5 \%$, indicating room for improvement with an effective anti-inflammatory treatment in these patients. Comparison of the demographics across the treatment groups did not show any significant imbalance.

There was no statistically significant difference between the change from baseline in trough FEV1 at week 6 for any dose compared with placebo, with the mean changes being $0 \mathrm{~mL}$ $(\mathrm{p}=0.51),-18 \mathrm{~mL}(\mathrm{p}=0.71)$ and $37 \mathrm{~mL}(\mathrm{p}=0.14)$ for the 0.1-mg, 0.4-mg and 1.0-mg doses, respectively.

The mean change from baseline in trough FEV1 in comparison with placebo at weeks 2 and 4 did not differ for either the 0.1$\mathrm{mg}$ or the 0.4-mg doses, although there was a statistically significant $(p=0.0001)$ change of $119 \mathrm{~mL}$ compared with placebo after 2 weeks in the 1.0-mg group (fig. 2). There were no statistically significant differences between any dose and placebo on any other secondary efficacy parameters at week 6; some of these findings are shown in table 2. As for FEV1, there was a tendency to a response after 2 weeks in the $1.0 \mathrm{mg}$ group on several parameters including FEV6, FVC, Mahler dyspnoea index and breathlessness on symptom scoring. This is illustrated for breathlessness in figure 3 and is supported by the TDI score of $>1$ point difference at week 2 for $1.0 \mathrm{mg}$

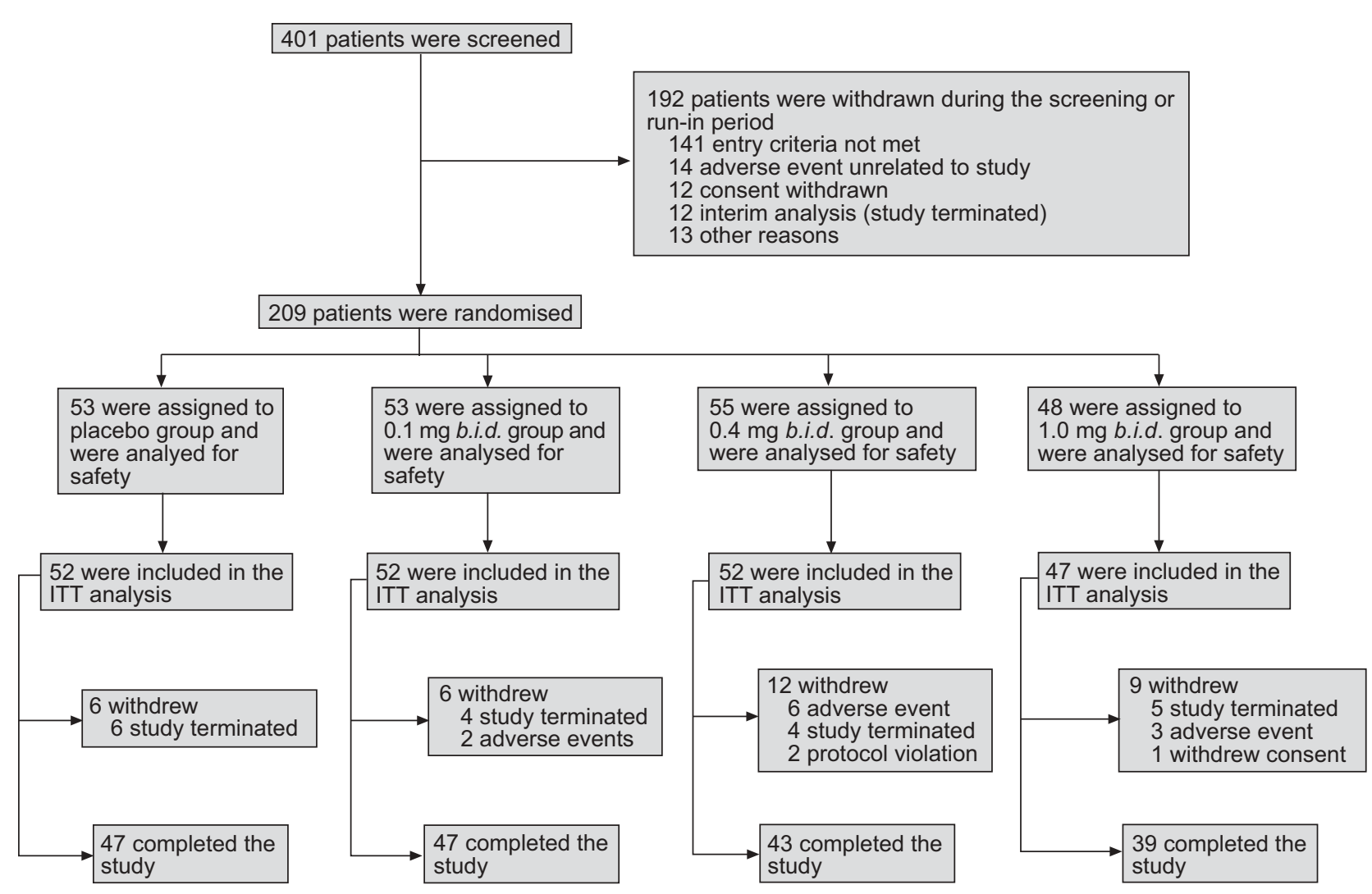

FIGURE 1. Consolidated Standards of Reporting Trials (CONSORT) diagram showing results of studies at clinical centres included in the present study. ITT: intention to treat. 


\begin{tabular}{|c|c|c|c|c|}
\hline TABLE 1 & $\begin{array}{l}\text { line charac } \\
\text { ded in the }\end{array}$ & $\begin{array}{l}\text { eristics for } \\
\text { resent stuc }\end{array}$ & e 209 pati & ints \\
\hline \multirow[t]{2}{*}{ Treatment } & \multicolumn{3}{|c|}{ UK-500,001 } & \multirow[t]{2}{*}{ Placebo } \\
\hline & $\begin{array}{l}0.1 \mathrm{mg} \\
\text { b.i.d. }\end{array}$ & $\begin{array}{c}0.4 \mathrm{mg} \\
\text { b.i.d. }\end{array}$ & $\begin{array}{l}1.0 \mathrm{mg} \\
\text { b.i.d. }\end{array}$ & \\
\hline Subjects n & 53 & 55 & 48 & 53 \\
\hline Male & $37(70)$ & $44(80)$ & $33(69)$ & $38(72)$ \\
\hline Age yrs & 65 (45-80) & 63 (43-78) & $62(46-79)$ & $65(45-78)$ \\
\hline Mean weight kg & 68.5 & 74.8 & 76.6 & 72.9 \\
\hline \multicolumn{5}{|l|}{ Smoking n } \\
\hline Smoker & 25 & 14 & 16 & 24 \\
\hline Ex-smoker & 28 & 41 & 32 & 29 \\
\hline FEV 1 L & $1.37 \pm 0.46$ & $1.45 \pm 0.47$ & $1.53 \pm 0.48$ & $1.43 \pm 0.49$ \\
\hline FEV $1 \%$ pred & $51.0 \pm 14.6$ & $49.3 \pm 12.3$ & $53.9 \pm 14.2$ & $52.2 \pm 14.2$ \\
\hline Reversibility \% & $16.2 \pm 15.0$ & $14.1 \pm 14.8$ & $14.0 \pm 14.1$ & $13.7 \pm 16.4$ \\
\hline
\end{tabular}

Data are presented as $n(\%)$, mean (range) and mean $\pm S D$, unless otherwise indicated. FEV1: forced expiratory volume in one second; \% pred: \% predicted.

compared with placebo. Further analyses were performed to assess the robustness of these conclusions, including analyses of patients who completed the study with no major protocol deviations, and analyses that examined whether smoking status, baseline reversibility or FEV1 \% pred affected the efficacy outcomes. All of these analyses were consistent with the results described above.

Compared with placebo, there was a slightly higher incidence of treatment-related AEs reported in the 0.4 and $1.0 \mathrm{mg}$ treatment groups; however, discontinuations due to AEs were similar across all groups. The most frequently occurring AEs are shown in table 3 . There were two serious AEs, as follows: one patient on $1.0 \mathrm{mg}$ of active drug experienced gastrointestinal effects requiring hospitalisation, and one patient on $0.4 \mathrm{mg}$ of active drug was admitted to hospital due to pneumonia. There were no differences in exacerbation rates across the treatment groups; $10-15 \%$ of subjects experienced an exacerbation during the study.

\section{DISCUSSION}

The present study of a novel inhaled PDE4 inhibitor showed no effect of treatment on spirometric measures and symptoms score in patients with moderate-to-severe COPD. Given the previous reports on efficacy of oral PDE4 inhibitors, the present finding is surprising and disappointing from the patients' outlook.

All negative trials should be interpreted with caution to ensure that study design and methodology have not led to incorrect conclusions [8]. The present study was adequately powered to detect clinically significant changes in trough FEV1, the observed SD $(157 \mathrm{~mL})$ was less than the expected SD $(173 \mathrm{~mL})$, and a number of sensitivity analyses confirmed the results from the primary analyses. There was no reason to believe that patients included in the present study would be less responsive than patients in previous trials of PDE4 inhibitors, given that they were of comparable baseline disease

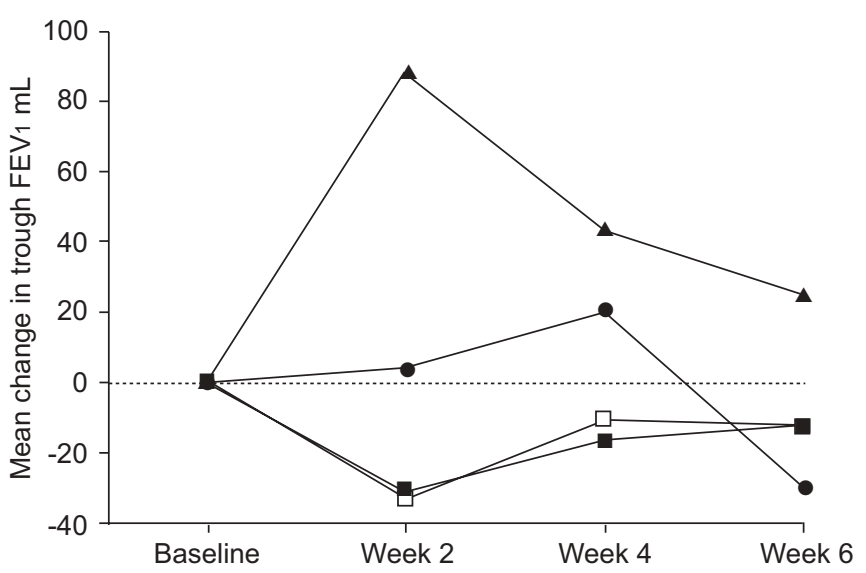

FIGURE 2. Mean change from baseline in trough forced expiratory volume in one second (FEV 1 ) at weeks 2, 4 and 6 for the four treatment groups: $\square$ : $0.1 \mathrm{mg}$ UK-500,001 b.i.d.; 0: 0.4 mg UK-500,001 b.i.d.; $\mathbf{\Delta}: 1.0$ mg UK-500,001 b.i.d.; : placebo

severity [2] and showed significant reversibility to bronchodilator therapy. In addition, the 6-week treatment duration was long enough to show clinical benefit in terms of improvements of trough FEV1 in studies of oral PDE4 inhibitors. Thus, the present authors do not think that the study methodology or design is to blame for the lack of effect on the primary or secondary efficacy variables at week 6 .

PDE4 inhibitors have been suggested as promising antiinflammatory agents targeting the inflammation characteristic of COPD [9]. Subsequently, roflumilast has been found to reduce the absolute number of neutrophils in induced sputum [10], but neither roflumilast nor cilomilast has been shown to change the percentage of neutrophils in sputum [10, 11]. A 12week study of cilomilast [11] showed a reduction of CD8+ and CD68+ T-lymphocyte subsets in bronchial biopsies. The only 1yr study published to date showed only modest changes as a result of treatment with roflumilast; i.e. a change in FEV1 of $39 \mathrm{~mL}$ and no statistically significant change in exacerbations,

\begin{tabular}{|c|c|c|c|c|}
\hline \multirow[t]{3}{*}{ TABLE 2} & \multicolumn{4}{|c|}{$\begin{array}{l}\text { Change from baseline in secondary effect } \\
\text { parameters }\end{array}$} \\
\hline & \multicolumn{3}{|c|}{ UK500,001 } & \multirow[t]{2}{*}{ Placebo } \\
\hline & $\begin{array}{l}0.1 \mathrm{mg} \\
\text { b.i.d. }\end{array}$ & $\begin{array}{l}0.4 \mathrm{mg} \\
\text { b.i.d. }\end{array}$ & $\begin{array}{l}1.0 \mathrm{mg} \\
\text { b.i.d. }\end{array}$ & \\
\hline \multicolumn{5}{|l|}{ Trough } \\
\hline FEV6 L & -0.050 & -0.040 & -0.007 & -0.015 \\
\hline FVC L & -0.095 & -0.065 & -0.025 & -0.023 \\
\hline IC L & 0.070 & 0.012 & -0.012 & 0.020 \\
\hline $\begin{array}{l}\text { Breathlessness } \\
\text { score }\end{array}$ & 0.066 & 0.037 & -0.062 & 0.014 \\
\hline Cough score & 0.100 & -0.077 & -0.120 & 0.080 \\
\hline Sputum score & 0.023 & 0.126 & 0.038 & -0.006 \\
\hline
\end{tabular}

FEV6: forced expiratory volume in six seconds; FVC: forced vital capacity; IC: inspiratory capacity. 


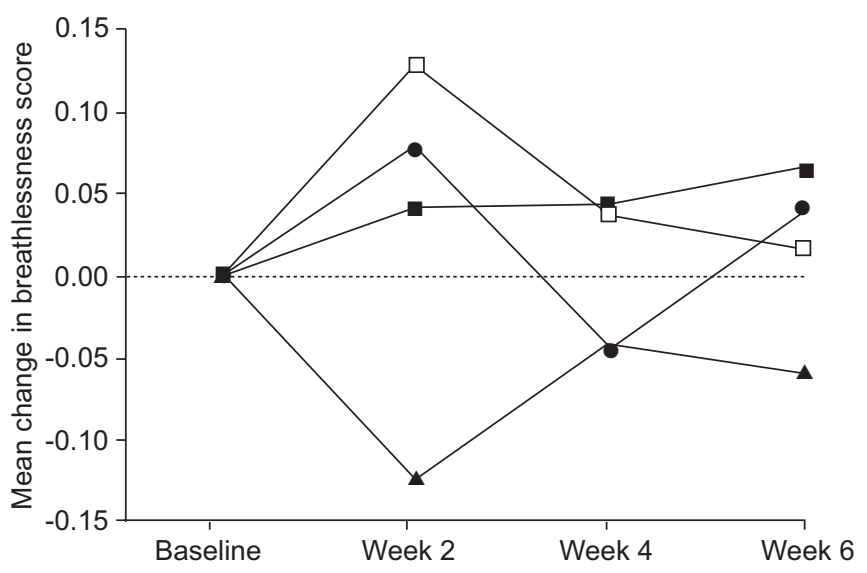

FIGURE 3. Mean change from baseline breathlessness score at weeks 2, 4 and 6 for the four treatment groups: $\mathbf{\square}: 0.1 \mathrm{mg}$ UK-500,001 b.i.d.; $\bullet: 0.4 \mathrm{mg}$ UK500,001 b.i.d.; $\mathbf{\Delta}: 1.0$ mg UK-500,001 b.i.d.; $\square$ : placebo.

although the latter was seen in a subgroup analysis of patients with very severe COPD [4]. This somewhat modest level of efficacy is consistent with a number of 26-week studies that were conducted with cilomilast, made publicly available via New Drug Application submissions to the Food and Drug Administration, and thoroughly reviewed by GIEMBYCZ [12]. However, in all clinical studies, treatment with PDE4 inhibitors has been associated with significant side-effects, in particular those affecting the gastrointestinal tract [2-4]. It seems unlikely that greater efficacy can be achieved with the existing oral PDE4 inhibitors by using higher doses, as maximal clinical tolerability seems to have been reached with current dosing [4, 12].

The present findings show a clear discrepancy with studies of oral PDE4 inhibitors in terms of the failure of UK-500,001 to show significant and maintained efficacy in moderate-tosevere COPD. The first explanation that comes to mind is that systemic PDE4 inhibition, and hence anti-inflammatory activity, is required to provide clinical benefit in this class of agents in COPD. However, the pre-clinical data do not support this explanation, as topical anti-inflammatory effects have been shown in animal models with inhaled UK-500,001 [5, 6]. In addition, an early (i.e. at 2 weeks) efficacy signal was detected in the primary efficacy parameter supported by improvements in some secondary efficacy parameters with the $1 \mathrm{mg}$ b.i.d. dosing group. Given that this improvement occurred only at the highest dose and was seen across a number of end-points, it would seem unlikely that this was due to a type 1 error (i.e. a false positive), suggesting a true efficacy signal with UK500,001 by the inhaled route at this time-point and dose. Finally, the adverse event profile in the present study indicates that there was also systemic PDE4-inhibitor action of inhaled UK-500,001. The early efficacy signal detected also seems to rule out a second possible explanation for the overall failure of the present study to detect significant and maintained efficacy, namely that UK-500,001 either did not reach areas of the diseased COPD lung containing active inflammation (e.g. due to mucus plugging and fixed airways obstruction leading to reduced airflow and ventilation, both well-described characteristics of COPD) in high enough concentrations or did reach

\begin{tabular}{|c|c|c|c|c|c|}
\hline \multirow[t]{3}{*}{ TABLE 3} & \multicolumn{5}{|c|}{$\begin{array}{l}\text { Adverse events occurring in }>5 \% \text { of patients in } \\
\text { any treatment group }\end{array}$} \\
\hline & & \multicolumn{3}{|c|}{ UK-500,001 } & \multirow[t]{2}{*}{ Placebo } \\
\hline & & $0.1 \mathrm{mg}$ & $0.4 \mathrm{mg}$ & $1.0 \mathrm{mg}$ & \\
\hline Subjects n & & 53 & 55 & 48 & \\
\hline \multicolumn{6}{|c|}{ Adverse events } \\
\hline Any & & $16(30.2)$ & 25 (45.5) & $17(35.4)$ & $23(43.4)$ \\
\hline Diarrhoea & & $2(3.8)$ & $4(7.3)$ & 3 (6.3) & $1(1.9)$ \\
\hline Headache & & $3(5.7)$ & $1(1.8)$ & $6(12.5)$ & $1(1.9)$ \\
\hline Respiratory & disorders & $6(11.3)$ & $10(18.2)$ & $5(10.4)$ & $7(13.2)$ \\
\hline
\end{tabular}

areas of inflammation in high enough concentrations but was not retained for a sufficient period of time to produce sustained activity. It is not possible to fully exclude this as an explanation, although it would seem surprising that a topically administered compound can exert systemic sideeffects, which were prolonged in phase I studies, without having any persistent effects in the respiratory system.

Given the early (i.e. 2-week) effect of $1.0 \mathrm{mg}$ b.i.d. UK-500,001 on both primary and secondary end-points, a more likely explanation for the lack of a maintained efficacy is the development of tachyphylaxis or tolerance on repeated dosing. Consistent with this interpretation is the clinical observation that the oral PDE4 inhibitors cilomilast and roflumilast seem to show greater levels of efficacy in shorter (i.e. 6 weeks $[2,3]$ ) as opposed to longer term (i.e. 26-52 weeks [4, 12]) clinical studies. To further support this explanation, there is the interesting pre-clinical observation that increasing levels of intracellular cyclic AMP (the main effect of PDE4 inhibition) may in itself lead to upregulation of PDE4 activity, either by phosphorylation or by altered gene transcription and translation [13], and could be part of the explanation for reduced inflammatory cell sensitivity to, for example, $\beta_{2}$-agonists on prolonged exposure [14].

In conclusion, the inhaled isotype-nonspecific, selective phosphodiesterase type-4 inhibitor UK-500,001 did not demonstrate efficacy at any dose, up to and including the maximum tolerated dose, in patients with moderate-to-severe chronic obstructive pulmonary disease. Given that the best of the oral isotype nonspecific agents described to date have shown only modest clinical benefit in chronic obstructive pulmonary disease in the absence of dose-limiting tolerance issues, an obvious attempt to improve the therapeutic index of this mechanism, with the potential to realise improved efficacy, has been to deliver a selective, but isotype nonspecific, phosphodiesterase type- 4 inhibitor by inhalation. This was the therapeutic rationale behind the progression of UK-500,001. Thus, the findings of the present study should lead to doubt about the future role of inhaled phosphodiesterase type- 4 inhibitors in chronic obstructive pulmonary disease, although it will require future studies with other inhaled phosphodiesterase type- 4 inhibitors to fully test this hypothesis. 


\section{ACKNOWLEDGEMENTS}

The investigators of the UK-500,001 Global Study Team are as follows. Argentina: J.C. Figuero Casas, A. Echazarreta, E. Giugno, O.E. Rizzo, M.C. De Salvo. Australia: P. Bardin, M. Phillips, J.P. Seale, P. Thompson. Canada: G.R. Bailey, D.S. Helmersen, K.J. Killian, D.E.M. O'Donnell. Chile: S. Chernilo, R. Sepúlveda. Croatia: F. Pavicic, N. Tudoric. Czech Republic: M. Kasl, V. Kolek, J. Musil. Hungary: E. Csanky, M. Namenyi, Z. Mark. Spain: R. Alvarez, P. De Lucas. UK: J. Vestbo.

\section{REFERENCES}

1 Rabe KF, Hurd S, Anzueto A, et al. Global strategy for the diagnosis, management, and prevention of chronic obstructive pulmonary disease: GOLD Executive Summary. Am J Respir Crit Care Med 2007; 176: 532-555.

2 Compton $\mathrm{CH}, \mathrm{Gubb} \mathrm{J}$, Nieman $\mathrm{R}$, et al. Cilomilast, a selective phosphodiesterase-4 inhibitor for treatment of patients with chronic obstructive pulmonary disease: a randomised, dose-ranging study. Lancet 2001; 358: 265-270.

3 Rabe KF, Bateman ED, O'Donnell D, Witte S, Bredenbröker D, Bethke TD. Roflumilast - an oral antiinflammatory treatment for chronic obstructive pulmonary disease: a randomised controlled trial. Lancet 2005; 366: 563-571.

4 Calverley PMA, Sanchez-Toril F, McIvor A, Teichmann P, Bredenbröker D, Fabbri LM. Effect of 1-year treatment with roflumilast in severe chronic obstructive pulmonary disease. Am J Respir Crit Care Med 2007; 176: 154-161.

5 Trevethick M, Banner K, Ballard S, et al. UK-500,001, a potent selective inhibitor of phosphodiesterase type 4 (PDE4) designed for inhaled delivery, inhibits $\mathrm{TNF} \alpha$,
MIP1 $\beta$ and $\mathrm{LTB}_{4}$ release from native human blood cells. Am J Respir Crit Care Med 2007; 175: Suppl. 1, A116.

6 Trevethick M, Philip J, Chaffe P, et al. In vivo profile of intratracheally delivered phosphodiesterase type 4 (PDE4) UK-500,001 inhibits bronchoconstriction and inflammation. Am J Respir Crit Care Med 2007; 175: Suppl. 1, A927.

7 American Thoracic Society, Standardization of spirometry, 1994 update. Am J Respir Crit Care Med 1995; 152: 11071136.

8 Freiman JA, Chalmers TC, Smith H, Kuebler RR. The importance of beta, the type II error and sample size in the design and interpretation of the randomized control trial. Survey of 71 "negative" trials. N Engl J Med 1978; 299: 690-694.

9 Vignola AM. PDE4 inhibitors in COPD - a more selective approach to treatment. Respir Med 2004; 98: 495-503.

10 Grootendorst DC, Gauw SA, Verhoosel RM, et al. Reduction in sputum neutrophil and eosinophil numbers by the PDE4 inhibitor roflumilast in patients with COPD. Thorax 2007; 62: 1081-1087.

11 Gamble E, Grootendorst DC, Brightling CE, et al. Antiinflammatory effects of the phosphodiesterase- 4 inhibitor cilomilast (ariflo) in chronic obstructive pulmonary disease. Am J Respir Crit Care Med 2003; 168: 976-982.

12 Giembycz MA. An update and appraisal of the cilomilast Phase III clinical development programme for chronic obstructive pulmonary disease. Br J Clin Pharmacol 2006; 62: 138-152.

13 Lugnier C. Cyclic nucleotide phosphodiesterase (PDE) superfamily: a new target for the development of specific therapeutic agents. Pharmacol Ther 2006; 109: 366-398.

14 Giembycz MA. Phosphodiesterase 4 and tolerance to $\beta_{2}$-agonists in asthma. Trends Pharmacol Sci 1996; 17: 331-336. 